\title{
Presurgical diagnostic difficulties in an asymptomatic patient with primary transitional cell carcinoma of the oviduct: case report
}

\author{
Nabil Abdalla, Anna Wnuk, Seweryn Trojanowski, Alicja Jakimiuk, Pawel Stanirowski, \\ Krzysztof Cendrowski, Wlodzimierz Sawicki
}

Department of Obstetrics, Gynecology and Gynecological Oncology, II Faculty of Medicine, Medical University of Warsaw, Poland

\begin{abstract}
Primary transitional cell carcinoma of the fallopian tube is a very rare condition. We present a case of a 70-year-old asymptomatic Caucasian patient with an irregular solid right adnexal mass of $67 \times 35 \times 59 \mathrm{~mm}$ which was discovered during routine ultrasound pelvic examination. There was no acoustic shadow and the patient did not feel pain during examination. No evidence of metastases or ascites was found by ultrasound. There was moderate vascularization of the mass. The mass was considered malignant according to the subjective assessment of the examiner. Serum level of CA125 was elevated to $519 \mathrm{U} / \mathrm{ml}$. The results of logistic regression model LR2 according to the International Ovarian Tumor Analysis (IOTA) group was 64.4\%, suggesting the malignant nature of the mass. The IOTA-ADNEX model showed $97 \%$ probability of malignancy, probably ( $85.5 \%)$ stage II-IV ovarian cancer. The risk of malignancy being borderline, stage I and metastatic was $0.6 \%, 3.9 \%$ and $7 \%$, respectively. Omitting CA125 in the IOTA-ADNEX model slightly decreased the probability of malignancy to $81.3 \%$, still most likely $(54.2 \%)$ stage II-IV ovarian cancer. The results of risk of malignancy indices RMI I-IV were 1557, 2076, 1557 and 2076, respectively, reflecting the malignant nature of the mass. The final diagnosis was transitional cell carcinoma of the fallopian tube, stage IIIc according to FIGO.
\end{abstract}

Key words: transitional cell carcinoma, fallopian tube, ultrasound.

\section{Introduction}

Fallopian tube malignancies are rare. Adenocarcinoma is the most common histological type of the tubal malignancy, representing $90 \%$ of them [1]. Primary carcinomas of the fallopian tube (PCFT) are the rarest neoplasms (0.1-1.6\%) of the female genital tract [2]. Primary transitional cell carcinoma (TCC) represents only $10 \%$ of all PCFT and it occurs mainly in postmenopausal patients [3]. The exact causes of this type of malignancy are not known. Some authors have linked this condition to BRCA1 and BRCA2 gene mutations, while multiparity can be a protective factor. Clinical presentation may include abdominal or pelvic mass, vaginal bleeding, abdominal pain and ascites [1]. The full Latzko triad of serosanguinous vaginal discharge or bleeding, pelvic pain and pelvic mass occurs in less than $15 \%$ of patients [2]. The most common symptom is atypical vaginal bleeding [4]. Owing to the lack of specific symptoms, PCFT is usually diagnosed in advanced cases [5].

\section{Case presentation}

A postmenopausal 70-year-old patient was admitted to our clinic because of a right adnexal mass dur- ing routine ultrasound examination. The patient was asymptomatic. She denied abdominal pain, bloating, gastrointestinal symptoms, urinary symptoms and vaginal bleeding. The patient had controlled hypertension and diabetes. She was a nonsmoker and had no family history of malignancies. She had delivered four times vaginally. She was amenorrheic for 22 years. Her past surgical history was unremarkable. She regularly underwent cytological cervical examination and the results were normal. The patient was in a good general health condition. Vital parameters were in the normal range. Her BMI was 32. Abdominal examination did not confirm abnormalities. Gynecological examination revealed a right-sided pelvic mass. The ultrasound examination was done according to terms and definitions of the International Ovarian Tumor Analysis (IOTA) group [6]. The mass was considered to be solid since more than $80 \%$ of the mass was solid. The borders of the mass were irregular, of $67 \times 35 \times 59 \mathrm{~mm}$ dimensions (Figs. 1 and 2). The vascularization of the mass was assessed as a score of 3, i.e. moderate flow (Fig. 3). There was no acoustic shadow and the patient did not feel pain during examination. No evidence of metastases or ascites was found by ultrasound. The mass was considered malignant according to the subjective assessment 


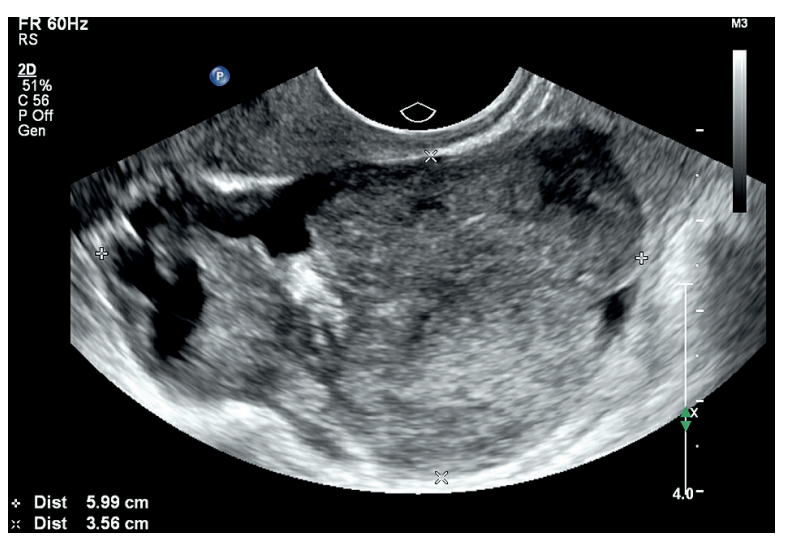

Fig 1. Gray scale ultrasound appearance of irregular unilateral solid adnexal mass

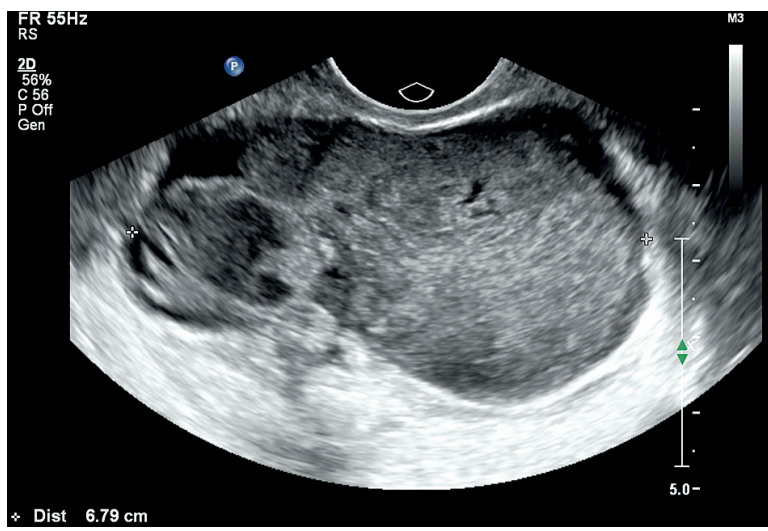

Fig. 2. Gray scale ultrasound showing largest diameter of the mass of $67 \mathrm{~mm}$

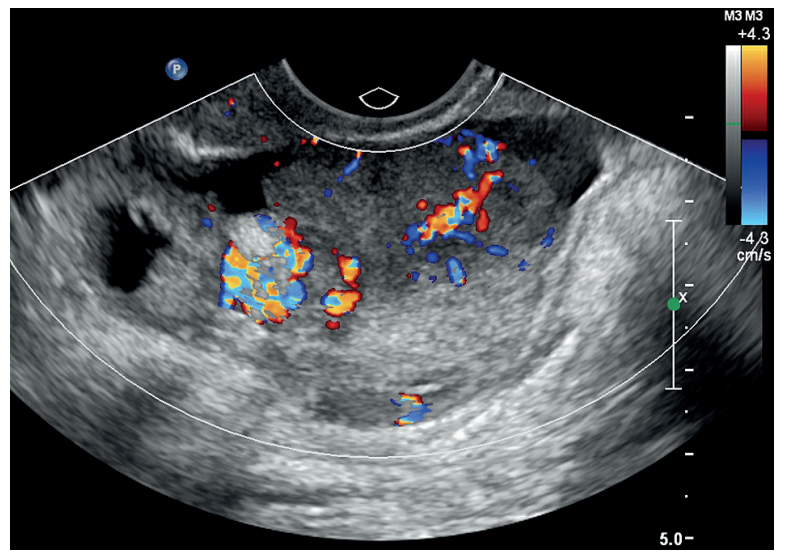

Fig. 3. Vascularization of the adnexal mass

of the examiner. Serum level of CA125 was elevated to $519 \mathrm{U} / \mathrm{ml}$. The serum level of $\alpha$-fetoprotein (AFP) and carcinoembryonic antigen (CEA) was within the normal range. The IOTA-ADNEX model showed $97 \%$ probability of malignancy, most likely (85.5\%) stage II-IV ovarian cancer. The risk of malignancy being borderline, stage I and metastatic was $0.6 \%, 3.9 \%$ and $7 \%$, respectively. Omitting CA125 in the IOTA-ADNEX model slightly de- creased the probability of malignancy to $81.3 \%$, still most likely (54.2\%) stage II-IV ovarian cancer, while the probability of borderline, stage I ovarian cancer and metastases was $2.1 \%, 10.9 \%$ and $14.1 \%$, respectively [7]. The result of logistic regression model LR2 according to IOTA was $64.4 \%$, suggesting the malignant nature of the mass [8]. The results of risk of malignancy indices RMI I-IV were 1557, 2076, 1557 and 2076 points respectively, reflecting the malignant nature of the mass [9]. Basic blood laboratory investigations, general urine examination and plain chest X-ray did not show abnormalities. The patient had a laparotomy where macroscopic inspection of the right adnexa revealed an enlarged, sausage-like oviduct, with an irregular surface and blue tinted color, filled with a fragile mass. Foci of tumor growth were seen on the outer surface of the oviduct. The ovary was in close proximity to the oviduct, without macroscopic changes. The left ovary and oviduct were without changes. The right pelvic lymph nodes were enlarged, forming a conglomerate of hard tissues in the right iliac fossa, the largest diameter being about $6 \mathrm{~cm}$. Other pelvic and abdominal structures were without macroscopic changes. The right adnexa were excised and sent for intraoperative histological assessment. The primary assessment indicated malignancy. The surgical operation was extended for staging, and the patient underwent hysterectomy, left adnexectomy, lymphadenectomy and omentectomy. The postoperative course was smooth and without complications. The final diagnosis was TCC of the fallopian tube, stage IIIc according to FIGO. Histological material expressed p63 (-) and p53 (-). The patient received 6 courses of chemotherapy in the form of carboplatin and paclitaxel. She tolerated the chemotherapy well. The patient has been in remission for 12 months following chemotherapy. The patient is still under strict oncologic follow-up.

\section{Discussion}

Presurgical diagnosis of fallopian tube cancer is difficult due to multiple factors. Rarity of the disease and non-specific clinical features contribute to these difficulties [4]. Primary malignant TCC of the oviduct can be 'a silent killer'. Our patient was asymptomatic, not representing any features of the known Latzko triad. The adnexal mass was picked up at routine ultrasound examination of the pelvis, and the malignancy was confirmed at advanced stage (IIIC according to FIGO). The patient refused to undergo tests of BRCA1 and BRCA2 gene mutations; however, the age of occurrence and negative family history may make the mutations less likely. Multiparity of the patient clearly did not prevent the disease.

Federman and Toker in 1973 described for the first time TCC of the oviduct [10]. A few cases of primary TCC of fallopian tubes have been reported in the literature 
$[4,11,12]$. Keepanasseril et al. reported two cases of this pathology. The first patient presented initially with lower abdominal pain and high grade fever of one month duration. Ultrasound showed low level echoes in the uterine cavity, suggesting pyometra. Ultrasound followup showed a unilateral adnexal mass with a normal range of CA125. Finally, stage I TCC of the oviduct was diagnosed. Fever subsided only after chemotherapy. The second patient presented at 55 years old because of postmenopausal bleeding and vaginal discharge. Endocervical tissues of the cervical curettage was suggestive of cervical cancer. TCC of the oviduct was confirmed after hysterectomy, bilateral salpingo-oophorectomy and pelvic lymphadenectomy [2].

Presurgical diagnosis of adnexal tumors is complex and involves clinical features, ultrasound parameters and tumor markers, as each parameter is not specific and may be absent [13]. A history of profuse intermittent vaginal discharge was the only symptom reported by Takeuchi et al., while lower abdominal pain and backache were the sole symptoms reported by Kim et al. $[4,11]$. The tumor marker CA125 is not necessarily elevated [4]. In our case these models were able to diagnose malignancy but without predicting the exact rare type of fallopian tube cancer. Final diagnosis of the tumor was made by histological assessment. Hu's criteria can be used for diagnosis of PCFT. These criteria include a tumor grossly within the fallopian tube, histological involvement of the tubal mucosa with papillary pattern, and evidence of transition between benign and malignant epithelium if the tubal wall is involved and the tube contains more tumor than the ovary or uterus [14].

Surgery combined with chemotherapy is the main treatment for PCFT. The presented patient is under strict follow-up. The study of Uehira et al. analyzed 21 cases of PCFT including 12 cases of TCC. The study revealed that TCC predominant tumors tended to relapse later than non-TCC-predominant tumors (mean, 31.2 vs 14.4 months after diagnosis), resulting in a significant difference in the 2-year disease-free survival rate [15].

\section{Conclusions}

Specific presurgical diagnosis of TCC of the oviduct is difficult due to the wide variety of clinical features, ultrasound parameters and nonspecific tumor markers. However, combining these various parameters may help in predicting malignancy even in asymptomatic patients. TCC should be kept in mind in the differential diagnosis of suspicious adnexal tumors.

\section{References}

1. Elsokkari I, Napaki S, Greening S, Aghmesheh M. Primary transitional cell carcinoma of the fallopian tube. J Obstet Gynaecol Res 2011; 37: 1767-1771.

2. Keepanasseril A, Bagga R, Saha SC, et al. Primary fallopian tube transitional cell carcinoma. J Obstet Gynaecol 2015; 35: 324-325.

3. Mardi K, Kaushal V. Primary Transitional Cell Carcinoma of the Fallopian Tube. Online J Health Allied Sci 2011; 10: 8.

4. Kim JW, Cho EM, Kim YT, Han JH. A case of primary transitional cell carcinoma of the fallopian tube. J Obstet Gynaecol Res 1999; 25: 321-326.

5. Ma Y, Duan W. Clinical and survival analysis of 36 cases of primary fallopian tube carcinoma. World J Surg Oncol 2014; 12: 311.

6. Timmerman D, Valentin L, Bourne TH, et al.; International Ovarian Tumor Analysis (IOTA) Group. Terms, definitions and measurements to describe the sonographic features of adnexal tumors: a consensus opinion from the International Ovarian Tumor Analysis (IOTA) Group. Ultrasound Obstet Gynecol 2000; 16: 500-505.

7. Van Calster B, Van Hoorde K, Valentin L, et al. Evaluating the risk of ovarian cancer before surgery using the ADNEX model to differentiate between benign, borderline, early and advanced stage invasive, and secondary metastatic tumours: prospective multicentre diagnostic study. BMJ 2014; 349: g5920.

8. Timmerman D, Testa AC, Bourne T, et al. Logistic regression model to distinguish between the benign and malignant adnexal mass before surgery: a multicenter study by the International Ovarian Tumor Analysis Group. J Clin Oncol 2005; 23: 8794-8801.

9. Yamamoto Y, Yamada R, Oguri H, et al. Comparison of four malignancy risk indices in the preoperative evaluation of patients with pelvic masses. Eur J Obstet Gynecol Reprod Biol 2009; 144: 163-167.

10. Federman Q, Toker C. Primary transitional cell tumor of the uterine adnexa. Am J Obstet Gynecol 1973; 115: 863-864.

11. Takeuchi S, Hirano H, Ichio T, et al. A case report: rare case of primary transitional cell carcinoma of the fallopian tube. J Obstet Gynaecol Res 1999; 25: 29-32.

12. Koshiyama M, Konishi I, Yoshida M, et al. Transitional cell carcinoma of the fallopian tube: a light and electron microscopic study. Int J Gynecol Pathol 1994; 13: 175-180.

13. Abdalla N, Winiarek J, Bachanek M, et al. Clinical, ultrasound parameters and tumor marker-based mathematical models and scoring systems in pre-surgical diagnosis of adnexal tumors. Ginekol Pol 2016; 87: 824-829.

14. Hu CY, Taymor ML, Hertig AT. Primary carcinoma of the fallopian tube. Am J Obstet Gynecol 1950; 59: 58-67.

15. Uehira K, Hashimoto H, Tsuneyoshi M, Enjoji M. Transitional cell carcinoma pattern in primary carcinoma of the fallopian tube. Cancer 1993; 72: $2447-2456$.

\section{Disclosure}

The authors report no conflict of interests. 